\title{
Results of a Quality Improvement Project Aimed at Eliminating Healthcare Waste by Changing Medical Resident Test Ordering Behavior
}

\author{
Sushilkumar Satish Gupta ${ }^{\mathrm{a}, \mathrm{i}}$, Radhika Voleti ${ }^{\mathrm{b}}$, Vimbai Nyemba ${ }^{\mathrm{c}}$, Selma Demir ${ }^{\mathrm{d}}$, Olaoluwatomi Lamikanra ${ }^{\mathrm{e}}$, \\ Nomsa Musemwa ${ }^{\mathrm{f}}$, Angela Saverimuthu ${ }^{\mathrm{e}}$, Kamaldeen Agoro ${ }^{\mathrm{e}}$, Robert D. Kalterg, Peter Homel ${ }^{\mathrm{h}}$, \\ Melvyn Hecht ${ }^{\mathrm{e}}$, Lawrence B. Wolfe, Edward K. Chapnick ${ }^{\mathrm{e}}$, Michael G. Kantrowitz ${ }^{\mathrm{e}}$, \\ Stephan L. Kamholz
}

\begin{abstract}
Background: In light of rising healthcare costs and evidence of inefficient use of medical resources, there is growing interest in reducing healthcare waste by clinicians. Unwarranted lab tests may lead to further tests, prolonged hospital stays, unnecessary referrals and procedures, patient discomfort, and iatrogenic anemia, resulting in significant economic and clinical effects. Blood tests are essential in guiding medical decisions, but they are also associated with significant financial and clinical costs. We designed a quality improvement study that attempted to decrease inappropriate ordering of laboratory tests while maintaining quality of care in a large residency program.
\end{abstract}

Methods: An algorithm outlining indications for complete blood count $(\mathrm{CBC})$, coagulation profile (PT/INR) and basic metabolic profile (BMP) was created by the study team. Data from 1,312 patients over a 3-month period in the pre-intervention phase and 1,255 patients during the selected intervention phase were analyzed. The primary endpoint was mortality rate and secondary endpoints were

Manuscript submitted September 22, 2017, accepted October 16, 2017

aDepartment of Pulmonary and Critical Care Medicine, Maimonides Medical Center, Brooklyn, NY, USA

${ }^{b}$ Department of Internal Medicine, Bay Health Kent General Hospital, Dover, DE, USA

'Department of Infectious Diseases, University of Maryland, Baltimore, MD, USA

dDepartment of Medicine, Baystate Medical Center, Springfield, MA, USA

eDepartment of Internal Medicine, Maimonides Medical Center, Brooklyn, NY, USA

fDepartment of Infectious Diseases, Penn State Hershey Medical Center, Hershey, PA, USA

gDepartment of Pathology and Laboratory Medicine, Maimonides Medical Center, Brooklyn, NY, USA

hBiostatistics Consultation Services, Department of Medicine, Maimonides Medical Center, Brooklyn, NY, USA

${ }^{\mathrm{i} C}$ Corresponding Author: Sushilkumar Satish Gupta, Department of Pulmonary and Critical Care Medicine, Maimonides Medical Center, 4802, 10th Avenue, Brooklyn, NY 11219, USA. Email: sugupta@maimonidesmed.org

doi: https://doi.org/10.14740/jocmr3210w length of stay and laboratory costs.

Results: There were significant decreases in the number of PT/INR orders (20.6\%), followed by BMP orders (12.4\%), and CBC orders $(9.3 \%)$. The mortality rate was $5.3 \%$ for the pre-intervention phase and $5.8 \%$ for the selected intervention phase, with a difference of $0.5 \%(\mathrm{P}=0.44)$.

Conclusion: Our approach leads to a decrease in costs, preventing unnecessary downstream testing, and improving patient experience. It also brought a mental discipline while ordering blood tests amongst residents.

Keywords: Daily blood draws; High value care; Healthcare waste; Resident education; Length of stay; Quality improvement

\section{Introduction}

Clinical laboratory tests are essential components of the diagnostic armamentarium available to physicians today. Results guide up to $70 \%$ of medical decisions [1]. Approximately $4-5$ billion laboratory tests are ordered yearly in the United States [2], contributing to about $4 \%$ of annual healthcare expenditure [3]. In light of rising healthcare costs, there is interest in reducing unnecessary and/or inappropriate ordering of laboratory tests [4]. Results of unwarranted lab tests prompt follow-up diagnostic tests, prolong length of hospital stay (LOS), and promote unnecessary referrals/procedures, patient discomfort, and iatrogenic anemia, all contributing to increased downstream costs $[5,6]$.

Various explanations exist for inappropriate lab ordering. In teaching hospitals, lab test ordering is usually performed by interns with attending physician oversight [7]. Interns sometimes order unnecessary lab tests because they may not be able to determine the ideal frequency for monitoring specific parameters. In addition, busy interns face time constraints that make it difficult to weigh the rationale for each individual test $[8,9]$. Furthermore, there is variability among attending physicians in determining the appropriateness of a test [10].

We designed a quality improvement project utilizing resi- 


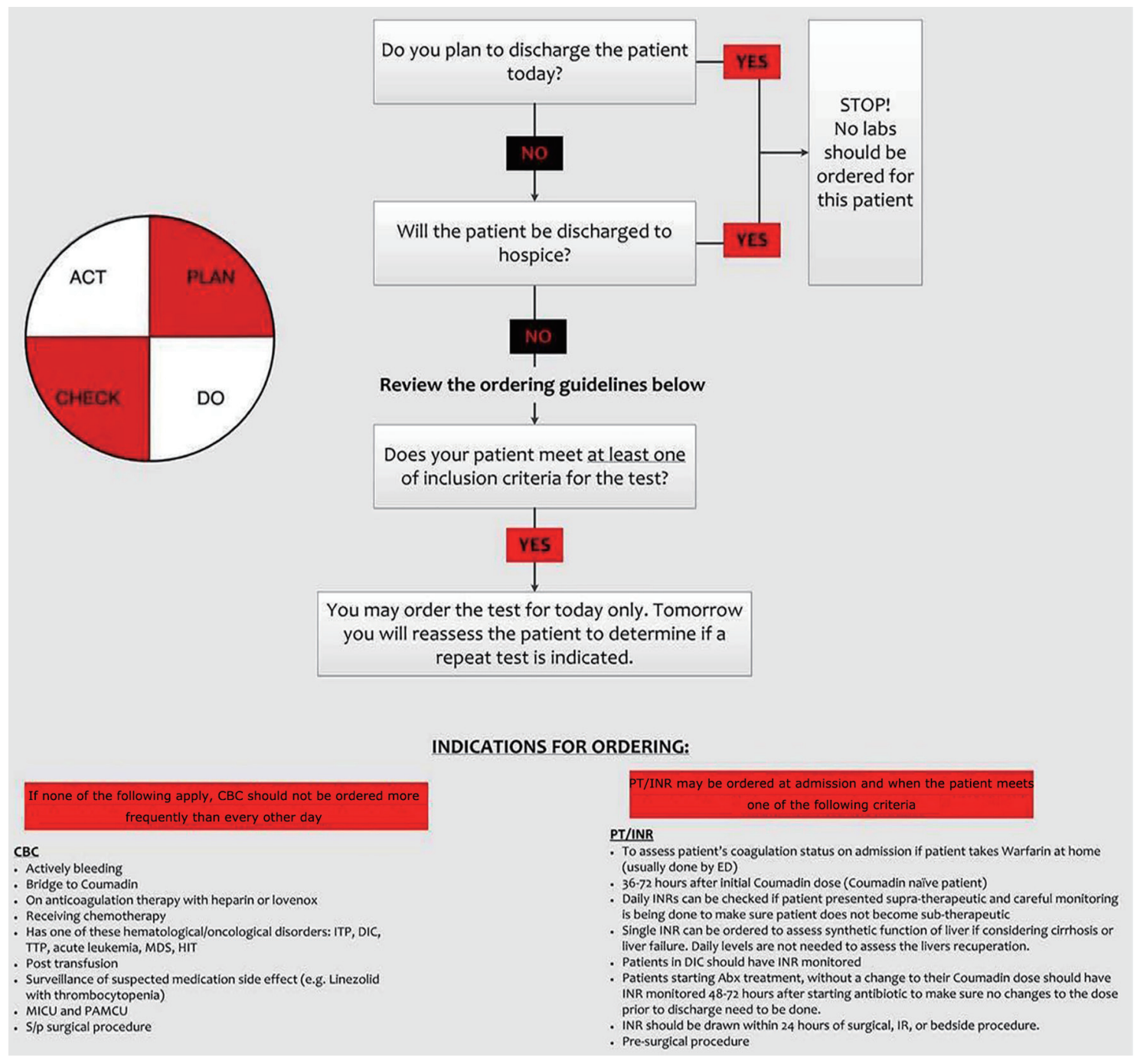

Figure 1. Algorithm utilized to guide ordering of laboratory investigations.

dent education and peer review in an attempt to decrease inappropriate ordering of laboratory tests on the medical in-patient units. We hypothesized that educating residents and encouraging utilization of a study-team-designed ordering algorithm, followed by frequent peer-review, would result in a decrease in the number of lab tests ordered, without clinically significant adverse effects, e.g., increases in mortality and the length of hospitalization.

\section{Methods}

\section{Site}

Maimonides Medical Center is a 711-bed tertiary hospital in Brooklyn, NY, with over 400 residents and fellows training in various specialties. The internal medicine inpatient teaching service includes interns and residents (supervised by faculty attending physicians) assigned to four unit based teams.

\section{Aims and objectives}

This quality improvement (QI) project was approved by the Institutional Review Board. We targeted the most commonly ordered lab tests: complete blood count (CBC), basic metabolic profile (BMP), and coagulation profile (PT/INR). The study population included patients admitted to internal medicine units from November 1, 2013 to April 1, 2015. Data collection included patients admitted from August 1, 2013 to October 31, 2013 , in order to assess ordering behavior in the pre-intervention phase for comparison purposes. An ordering algorithm was formulated to guide residents ordering of lab tests (Fig. 1).

\section{Intervention}

The study team initiated a multi-pronged intervention on October 31, 2013, beginning with lectures for the residents. Lectures outlined the high-value cost conscious care approach to 


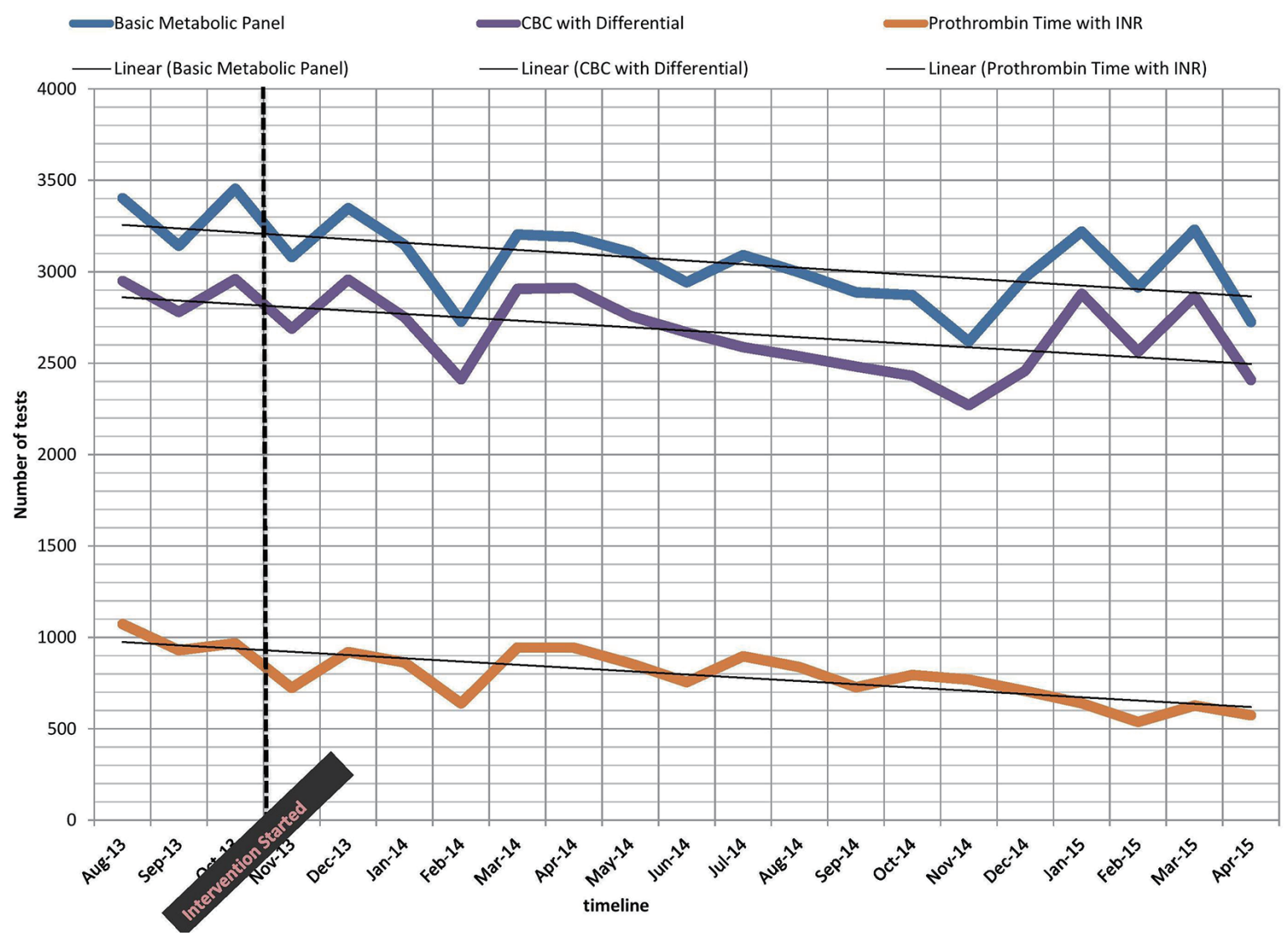

Figure 2. Graph demonstrating the decline in tests ordered during study period. The intervention line highlights the start of the project.

lab ordering and introduced the study-team-designed ordering algorithm. Thereafter, the study team formed subgroups assigned to each inpatient team which conducted regular surveillance and assessed progress. The hospital's clinical analytics department provided patient demographic data, and the finance department provided details about cost of labs.

\section{Statistical analysis}

The "pre-intervention phase" was the 3 months preceding the start of the intervention (August 1, 2013 to October 31, 2013). The same months during the intervention period (August 1, 2014 to October 31, 2014) were selected for comparison to attempt to minimize seasonal changes on variables (defined as the "selected intervention phase"). The primary outcome was a comparison of mortality before and after the introduction of the QI project. We calculated a 5.3\% mortality rate, from the data provided by the clinical and quality analytic team in the pre-intervention phase and estimated that the mortality rate during the selected intervention phase would be $\pm 2 \%$. A twogroup Chi-square test was used to determine clinically significant differences in mortality rates between the pre-intervention and selected intervention phases. Secondary endpoints included changes in laboratory costs and changes in LOS. A sample of 2,185 patients/period was estimated to provide at least $80 \%$ power to detect any difference in rate of mortality greater than $2 \%$ with alpha of 0.05 . We estimated that we needed to collect data for approximately 5,000 patients in order to have a reliable estimate of mortality rate.

\section{Results}

During the 21-month study period, 9,156 patients were admitted to the hospital units (excluding intensive care units) staffed by medical residents: $1,314(14 \%)$ during the pre-intervention phase from August to October 2013, and 7,842 (86\%) patients during the entire intervention period from November 2013 to April 2015. The actual mortality rate was $5.3 \%$ for the pre-intervention phase, and $5.8 \%$ for the selected intervention phase $(\mathrm{P}=0.44)$. The average LOS for the pre-intervention phase was 5.61 days, while LOS for the selected intervention phase was 6.45 days, with a difference of 0.85 day.

A comparison of the number of laboratory tests between the pre-intervention phase and selected intervention phase showed a decrease in all types of tests. CBC orders were decreased by $9.3 \%$ (9,763 to 8,854$)$, and BNP orders by $12.4 \%$ $(9,999$ to 8,755$)$. The most significant decrease was seen in PT/ INR orders, which was decreased by $20.6 \%(2,969$ to 2,358$)$. 
Table 1. Cost Comparison Between the Pre-Intervention and Selected Intervention Phases

\begin{tabular}{|c|c|c|c|c|c|c|c|}
\hline & $\begin{array}{l}\text { Cost of CBC } \\
\text { with and with- } \\
\text { out differential }\end{array}$ & $\begin{array}{l}\text { Number } \\
\text { of orders }\end{array}$ & Cost of BMP & $\begin{array}{l}\text { Number of } \\
\text { BMP orders }\end{array}$ & $\begin{array}{l}\text { Cost of } \\
\text { PT/INR }\end{array}$ & $\begin{array}{l}\text { Number } \\
\text { of PT/INR } \\
\text { orders }\end{array}$ & $\begin{array}{l}\text { Total cost } \\
(\mathrm{CBC}, \mathrm{BMP} \text {, } \\
\text { and } \mathrm{PT})\end{array}$ \\
\hline August, September, October 2013 & $\$ 70,999.7$ & 9,763 & $\$ 82,691.73$ & 9,999 & $\$ 11,282.2$ & 2,969 & $\$ 164,973.63$ \\
\hline August, September, October 2014 & $\$ 62,209.1$ & 8,854 & $\$ 72,403.85$ & 8,755 & $\$ 8,960.4$ & 2,358 & $\$ 143,573.35$ \\
\hline$\$$ decrease & $\$ 8,790.7$ & - & $\$ 10,287.88$ & - & $\$ 2,321.8$ & - & $\$ 21,400.28$ \\
\hline$\%$ decrease & 12.38 & 9.3 & 12.44 & 12.4 & 20.57 & 20.6 & 12.97 \\
\hline
\end{tabular}

Summary data for the number of laboratory tests ordered during the entire period (Fig. 2) revealed a similar trend, with decrease in the number of all types of laboratory tests. The 3-month total cost for CBC, BMP, and PT/INR was $\$ 164,973$ for the pre-intervention phase, versus $\$ 143,573$ for the selected intervention phase, resulting in a $\$ 21,400$ cost saving (Table $1)$. Extrapolating to a full year yielded an estimated $\$ 85,600$ savings per year.

\section{Discussion}

The primary objective of the study was to implement high-value cost conscious care without negatively impacting the quality of care. The advantages of reducing unnecessary lab test orders included the elimination of false positive results that may lead to unnecessary downstream testing. Benefits to the patient included elimination of painful phlebotomy attempts and associated complications such as hematomas, superficial vein thrombosis, and skin infections, leading to improved patient experience. The elimination of excessive venipunctures was perhaps of greatest benefit in hospice patients, since diagnostic lab testing did not necessarily improve outcomes and caused discomfort.

Our study supported the hypothesis that decreasing the frequency and number of laboratory investigations did not produce a significant difference in mortality. Our study demonstrated a $9.3 \%$ reduction in the number of BMPs ordered, $12.4 \%$ reduction in $\mathrm{CBC}$ tests and $20.6 \%$ decrease in PT/INR tests, leading to a cost savings of $\$ 21,400$.

We observed a small increase in LOS of 0.8 day; however, this difference was less than 1 day, which was the pre-established limit to define clinically significant change. We identified multiple factors that may have accounted for this increase in average LOS. In October 2013, Centers for Medicare and Medicaid Services (CMS) implemented the two-midnight rule, dictating that inpatient admissions of Medicare and Medicaid patients would be only reimbursed if LOS spanned at least two midnights. This favored admission of patients likely to require longer stays, and prompted emergency room (ER) discharge of those likely to require short inpatient stays. A reduction in inpatient bed capacity (August 2014) may have impacted the increase in LOS in the selected intervention phase. The closure of approximately 30 beds led to an increase in the duration of ER stay for admitted patients prior to bed assignment, since increased ER dwell time is an independent risk factor for prolonged LOS [11].
Several studies have shown that overutilization of laboratory tests is responsible for major costs in healthcare expenditure in teaching hospitals and a reduction in ordering these tests leads to considerable healthcare savings [1], which was one of the objectives of our study.

\section{Limitations}

We were unable to obtain data on individual patients with International Classification of Diseases, ninth revision (ICD-9) diagnoses, and thus could not perform subgroup analysis for change in LOS. Hawthorne effect may have played a role in the reduction in lab test ordering observed in our study. Study team members who performed peer-review were required to contact the interns that had ordered inappropriate lab tests and provide re-education.

\section{Conclusion}

Healthcare waste is a multi-billion-dollar problem and every healthcare provider must carefully weigh costs (including downstream costs), harms, and benefits and order only those interventions that add value to a patient's care. We advocate the use of evidence-based guidelines and decision support tools to practice high-value care.

\section{Conflict of Interest}

The authors declare that there is no conflict of interest.

\section{Funding}

None.

\section{Prior Presentations}

The paper was presented as a poster, at the Eighth Annual Integrating Quality Meeting of Association of American Medical College (AAMC), held on June 9, 2016 at Chicago, IL. The paper was highly appreciated by the viewers and program directors of the other hospitals. 


\section{References}

1. Bissell MG. Pending laboratory tests and the hospital discharge summary in patients discharged to sub-acute care. J Gen Intern Med. 2011 Apr;26(4):393-398.

2. Zhi M, Ding EL, Theisen-Toupal J, Whelan J, Arnaout R. The landscape of inappropriate laboratory testing: a 15year meta-analysis. PLoS One. 2013;8(11):e78962.

3. Warren JS. Laboratory test utilization program: structure and impact in a large academic medical center. Am J Clin Pathol. 2013;139(3):289-297.

4. Berwick DM, Hackbarth AD. Eliminating waste in US health care. JAMA. 2012;307(14):1513-1516.

5. Krasowski MD, Chudzik D, Dolezal A, Steussy B, Gailey MP, Koch B, Kilborn SB, et al. Promoting improved utilization of laboratory testing through changes in an electronic medical record: experience at an academic medical center. BMC Med Inform Decis Mak. 2015;15:11.

6. Thavendiranathan P, Bagai A, Ebidia A, Detsky AS, Choudhry NK. Do blood tests cause anemia in hospitalized patients? The effect of diagnostic phlebotomy on hemoglobin and hematocrit levels. J Gen Intern Med. 2005;20(6):520-524.

7. Vidyarthi AR, Hamill T, Green AL, Rosenbluth G, Baron RB. Changing resident test ordering behavior: a multilevel intervention to decrease laboratory utilization at an academic medical center. Am J Med Qual. 2015;30(1):8187.

8. May TA, Clancy M, Critchfield J, Ebeling F, Enriquez A, Gallagher C, Genevro J, et al. Reducing unnecessary inpatient laboratory testing in a teaching hospital. Am J Clin Pathol. 2006;126(2):200-206.

9. Griffith $\mathrm{CH}, 3 \mathrm{rd}$, Rich EC, Hillson SD, Wilson JF. Internal medicine residency training and outcomes. J Gen Intern Med. 1997;12(6):390-396.

10. Hauser RG, Shirts BH. Do we now know what inappropriate laboratory utilization is? An expanded systematic review of laboratory clinical audits. Am J Clin Pathol. 2014;141(6):774-783.

11. Liew D, Liew D, Kennedy MP. Emergency department length of stay independently predicts excess inpatient length of stay. Med J Aust. 2003;179(10):524-526. 